\title{
UNCERTAINTY IN DETERMINING THE PARAMETERS OF THE SURFACE DEFORMATION MODEL
}

\author{
Wojciech GRUSZCZYŃSKI, Zygmunt NIEDOJADLO and Dawid MROCHEŃ*
}

\author{
AGH University of Science and Technology, Faculty of Mining Surveying and Environmental Engineering, \\ Al. Mickiewicza 30, 30-059 Cracow, Poland \\ *Corresponding author's e-mail: dawmro@agh.edu.pl
}

\begin{tabular}{|c|c|}
\hline ARTICLE INFO & ABSTRACT \\
\hline Article history: & \multirow{6}{*}{$\begin{array}{l}\text { Predicting surface deformations caused by underground mining is an issue of significance both } \\
\text { for the safety of overlaying facilities and for economic purposes. There are many different } \\
\text { models for predicting the impact of underground mining on the land surface. One of them is the } \\
\text { Knothe model commonly used in Poland and in the world. The paper presents two methods of } \\
\text { estimating Knothe model parameters uncertainty. The parallel application of two methods } \\
\text { enables the mutual verification of the results obtained and the identification of the potential } \\
\text { errors and their sources in the case of any discrepancies. The first method is based on the so- } \\
\text { called law of propagation of uncertainty, which in essence is the approximation based on the } \\
\text { first-order Taylor series expansion. The second presented method is based on the Monte Carlo } \\
\text { simulation. }\end{array}$} \\
\hline Received 5 February 2019 & \\
\hline Accepted 15 April 2019 & \\
\hline Available online 26 April 2019 & \\
\hline Keywords: & \\
\hline $\begin{array}{l}\text { Mining deformations } \\
\text { Monte Carlo method } \\
\text { Uncertainty estimation }\end{array}$ & \\
\hline
\end{tabular}

\section{INTRODUCTION}

Predicting surface deformations caused by underground mining is an issue of significance both for the safety of overlaying facilities and for economic purposes.

There are many different models for predicting the impact of underground mining on the land surface (Ghabraie et al., 2017; Gruszczyński, 2007; Karmis et al., 1987; Ren et al., 2014; Tajduś, 2009; Whittaker and Reddish, 1989). One of them is the Knothe (Knothe, 1957) model commonly used in Poland and in the world (Byrnes, 2003; Doležalová et al., 2009; Karmis et al., 1990; Luo and Cheng, 2009). Based on this method, considerations were made to estimate the uncertainty of deformation model parameters.

The values of the parameters assumed for computations are crucial for the results of all prediction models. Their values are assumed on the basis of previous experiences or the on-going surveys of surface deformations for any pending exploitation (Kowalski, 2007; Hejmanowski and Malinowska, 2016).

Uncertainty of the deformation indicator prediction is strictly correlated with uncertainty of the determined parameters of a deformation model. It depends on the source of these parameters and is usually lower when determining the parameters based on on-going surveys for the exploitation in progress (Gruszczyński et al., 2018).
The subject-matter of this paper is to analyze this very case, whereas its purpose is to compare two methods of estimating model parameter uncertainty. The application of two methods is also aimed at the verification of the results.

In addition, this article provides the answer to three questions of significance for the discussed context:

- What is the uncertainty in determining the parameters and how does it change along with exploitation progress?

- What is the dispersion of the estimated values of parameter uncertainties?

- What is the accuracy of determination of the model parameters when using data obtained by unmanned aerial vehicles (UAV) by using photogrammetry or airborne laser scanning (ALS)?

The presented results are continuations of the research presented in our previous paper (Gruszczyński et al., 2018).

\section{MATERIALS AND METHODS \\ 2.1. METHODS OF ESTIMATION OF MODEL PARAMETER UNCERTAINTY}

The opportunity to estimate the parameter uncertainty and correlation between their values is of significance for the evaluating the accuracy of 
a prediction. The studies compare two methods used for this purpose - i.e. the law of propagation of uncertainty (Gruszczyński et al., 2018; Kwinta, 2011; Mierzejowska, 2014) and the Monte Carlo method (Kwinta, 2010). The proposed methods provide different options and different computing load.

The Monte Carlo method (MC) usually requires greater effort and can generate any statistics for deformation indicators. This method can also be used with high values of parameter uncertainty and strongly non-linear functions. In Monte Carlo method the subsidences calculated by using model with the parameter values estimated by the least squares method are considered as conditional average values (averages for a given point and given course of exploitation).

These values are then added up along with white noise with a zero mean and no correlation between its values. The uncertainty of the subsidences is assumed from the solution of the equation system, whereas the noise distribution is assumed as normal.

The disturbed values of subsidences are fitted with the model and the values of its parameters are determined. This procedure is repeated several times, each time giving slightly different values of the model parameters.

Furthermore, the studies - in each iteration (single model matching) - calculate the uncertainties and correlations of parameters based on LPU. The values of parameters, uncertainties and correlations collected in such a manner can be treated as a random sample and used as a basis to estimate, for instance, the values of parameter uncertainty and Pearson correlation coefficient between them. This method also enables the parameter probability density function to be estimated as well as the dispersion to be evaluated, within which the LPU estimates the uncertainty values (uncertainty of parameter uncertainty) and correlation values (uncertainty of coefficient of correlation between the parameters).

Compared to this method, the application of the so-called law of propagation of uncertainty implies certain limitations. Firstly, it enables only the standard uncertainty of the function value to be estimated on the basis of knowing the standard uncertainty of its arguments rather than by generating any statistics. This law, in the version applied in the studies, provides accurate results only for relatively minor uncertainties of the model parameters. In addition, the quality of estimation is dependent here on the shape of the approximated function - i.e. the closer to the linear function, the higher the quality of estimation of uncertainty.

\subsection{EXPERIMENT SETUP}

The data (subsidences) applied to the studies were simulated by using the Knothe model. The flow of the simulated progress of exploitation generating surface deformations was compliant with the scheme presented in Figure 1.

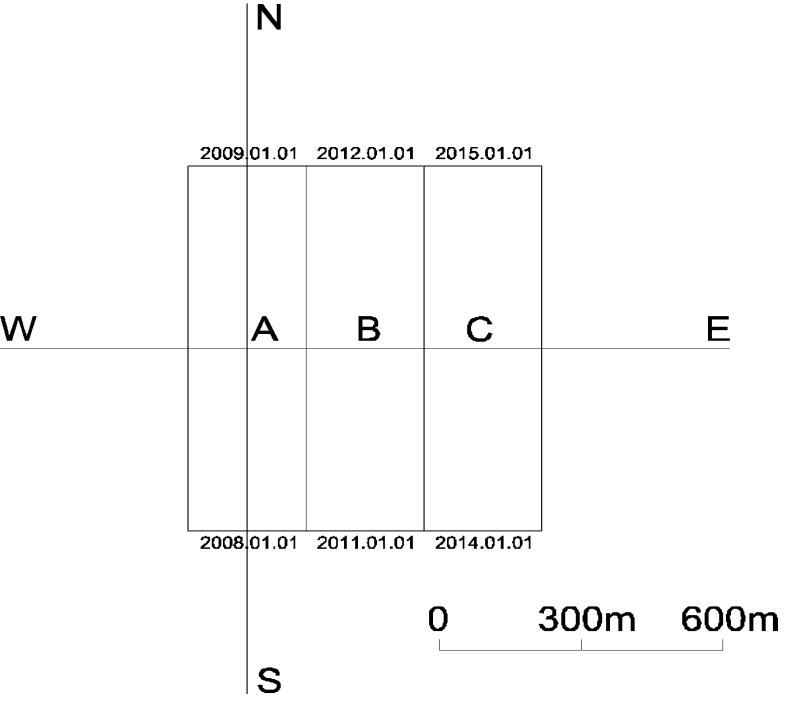

Fig. 1 Flow of simulated exploitation and location of observational lines.

The simulated exploitation covered three longwalls conducted by collapsing the roof at a depth of $600 \mathrm{~m}$, with the height of the exploitation deposit at $2.5 \mathrm{~m}$, with a width of $250 \mathrm{~m}$ and a length of $900 \mathrm{~m}$ each. Exploitation progressed from the longwall located the furthest to the west (marked in the Figure 1 as A), via the one located in the middle (longwall B) to the one located to the east (longwall C). The simulated values of subsidences were determined after the exploitation of each subsequent longwall for an amount of time sufficient to omit the influence of time on the flow of the subsidence - i.e. upon the manifestation of the complete influence of exploitation on longwalls A, B and C, respectively. The calculated subsidence values correspond to the (true) average subsidence conditions caused by exploitation of longwall A (hereinafter referred to as exploitation stage A), longwalls A and B (exploitation stage B) and longwalls A, B and C (exploitation stage $\mathrm{C}$ ), respectively.

The subsidences were calculated for the points located at the lines crossing the centre of longwall A and marked in Figure 1 as NS and WE, respectively, as well in the regular grid. Both the lines and the point grid exceeded the wall outline by $400 \mathrm{~m}$, so that the calculated subsidences would cover a complete range of subsidences caused by the simulated exploitation. The points along the observation lines were located every $25 \mathrm{~m}$. This corresponds, in approximation, to the distances between the points on the observation lines from the area of the Upper Silesian Coal Basin (USCB). The NS observation line consist of 69 points and the second one (WE) has 63 points.

For the purposes of the calculations, the distance between the nodes of the grid simulating the data from the UAV surveys was $10 \mathrm{~m}$. With such a grid density, the calculations were performed for more than 26 thousand points. Calculating the subsidences within 
Table 1 Parameters from the USCB region.

\begin{tabular}{ll}
\hline Parameter & Mean value \\
\hline$a$ & 0.80 \\
$\tan \beta$ & 1.92 \\
$p=k \cdot H$ & $0.1 \mathrm{H}(=60 \mathrm{~m})$ \\
\hline
\end{tabular}

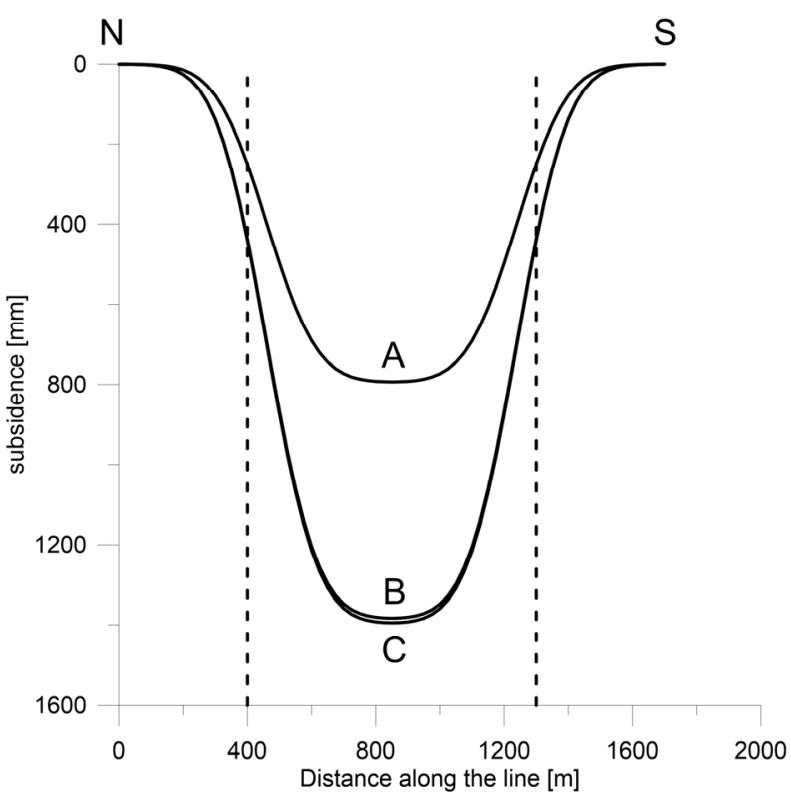

Table 2 The adopted values of the random scatter of simulated subsidences.

\begin{tabular}{lc}
\hline Cases & $\sigma_{s}[\mathrm{~mm}]$ \\
\hline Lines NS and WE, stage A & \pm 16 \\
Lines NS and WE, stage B & \pm 35 \\
Lines NS and WE, stage C & \pm 39 \\
Grid, stage A, B, C & \pm 150 \\
\hline
\end{tabular}

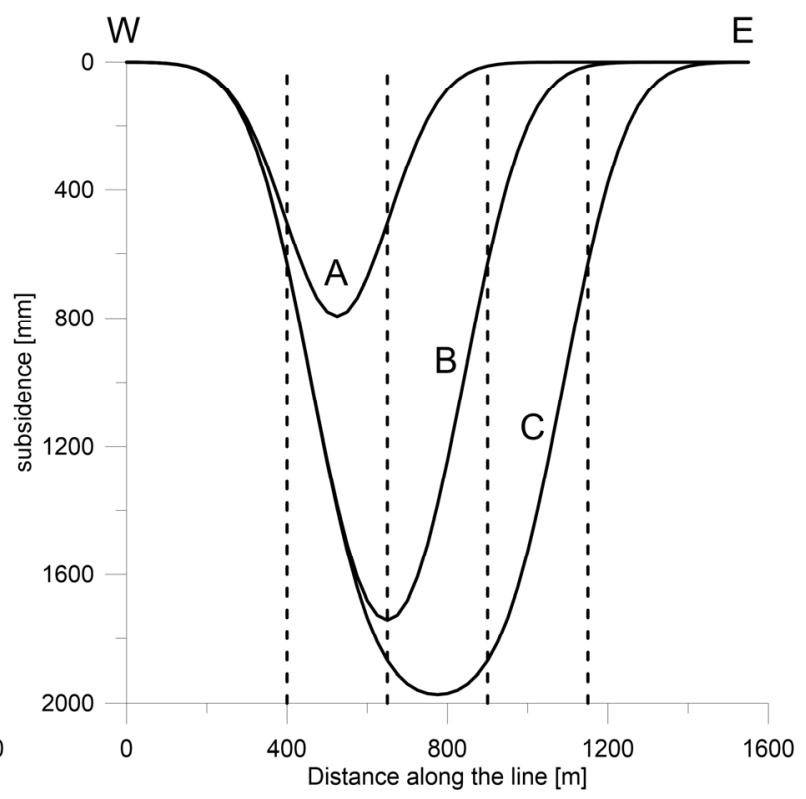

Fig. 2 Subsidence along the NS and WE lines for the subsequent exploitation stages.

the grid aims to simulate the surveys performed by using UAV with photogrammetry or ALS. In such a case, the points would be located with a much greater density (Gruszczyński at al., 2017; Jiang and Jiang, 2017; Lizarazo et al., 2017; Lucieer et al., 2014; Tsai and Lin, 2017), although it was stated that the chosen density was sufficient to reflect the properties of these method and assure a reasonable calculation time.

The subsidences were modelled on the model parameters average (Kowalski, 2007) for collapsed roof exploitation carried out in the area of the USCB. These values are presented in Table 1, where $a$ is the exploitation coefficient, $\tan \beta$ is a parameter characterizing the rock mass and its geo-mechanical properties, $p$ is an operating rim which is the function of factor of the operating rim parameter $k$ and exploitation depth $H$.

The subsidence (their conditional average values i.e. systematic factor) along the NS and WE lines for the subsequent exploitation stages are presented in Figure 2.

Random scatter for subsidences determined on the NS and WE lines were matched in accordance with the results of the Kowalski (Kowalski, 2007) and Stoch (Stoch, 2005) studies as $2 \%$ of maximum subsidence for a given exploitation status (Table 2). Random scatter for the points located on the grid nodes was selected on the basis of experiments (Gruszczyński et al., 2017) by using UAV and LIDAR tech sheet systems assembled on the UAV. For the grid points, it was assumed that the amplitude would not depend on the exploitation status, since in such a case a dominant role will be played by measurement uncertainties rather than random scatter of the phenomenon.

The experiments were repeated 1,000 times. The optimum values of the parameters were determined for each iteration. Any uncertainties and correlations between the parameters were estimated on the basis of the law of propagation of uncertainty.

\section{RESULTS AND DISCUSSION}

The results of the calculations were analyzed in relation to the questions formulated in the introduction to the paper. The uncertainties in determining the parameter values along with the progress of exploitation (stages A, B and C) for all the analyzed cases - i.e. for the NS line, WE line, both lines and for grid (UAV) - are presented in Figure 3. In all these graphs, the vertical axes are broken due to much a greater uncertainty of parameters estimated on the basis of series A for the WE line compared with the other cases. This special case will be analyzed further in the paper. 

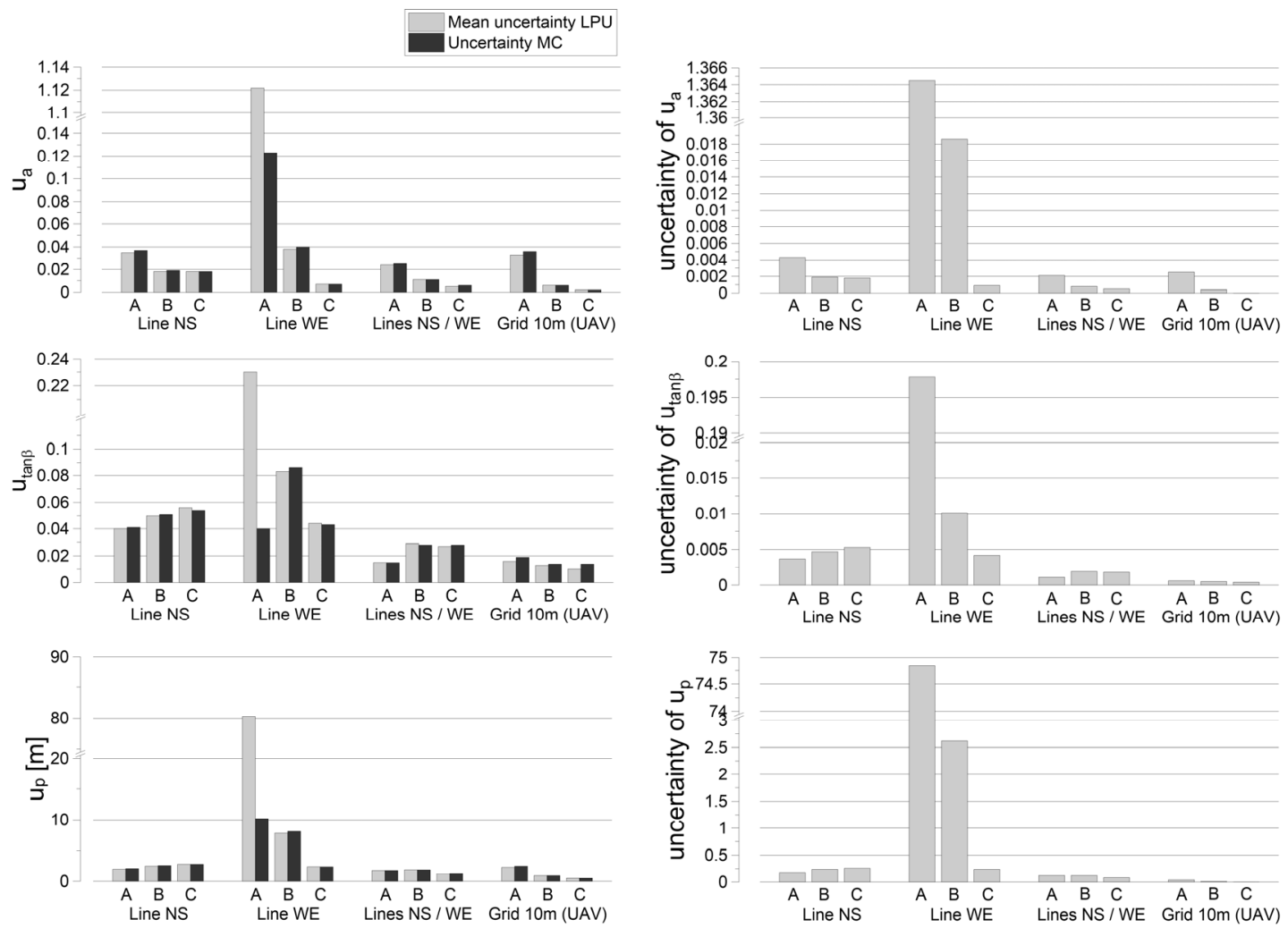

Fig. 3 Uncertainty and the uncertainty of the uncertainty of estimating the model parameters depending on the source of observation and exploitation stage.
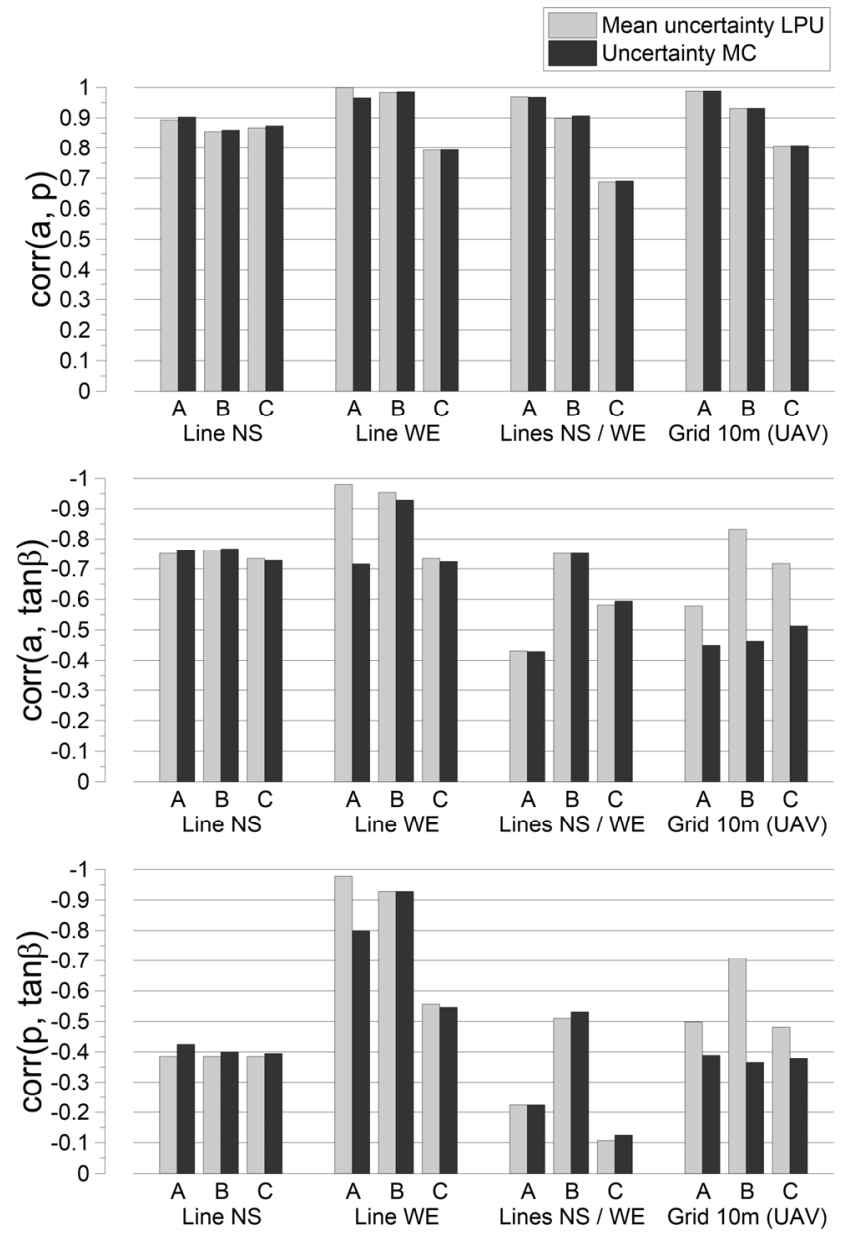

Fig. 4 Correlations between the model parameters. 

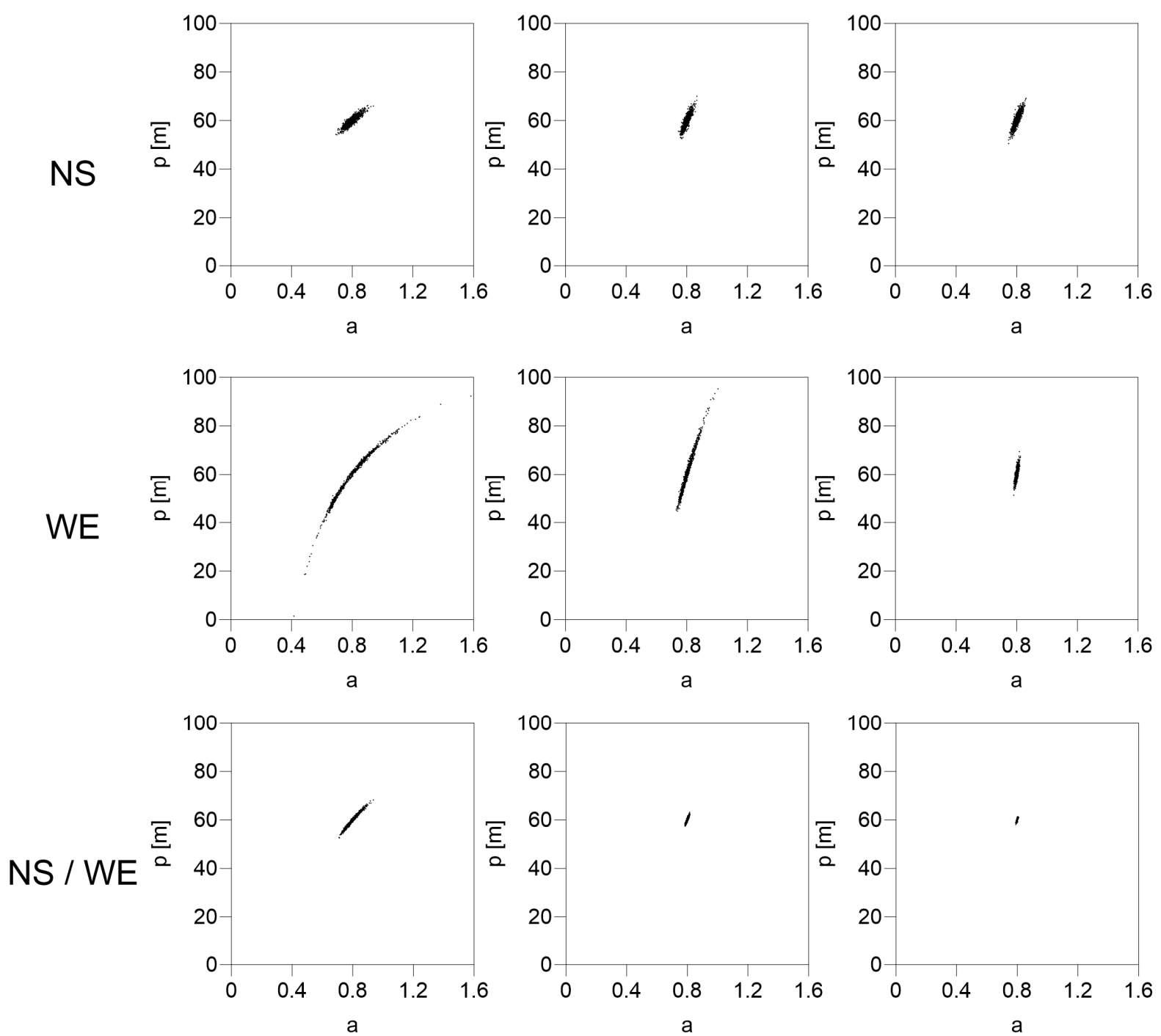

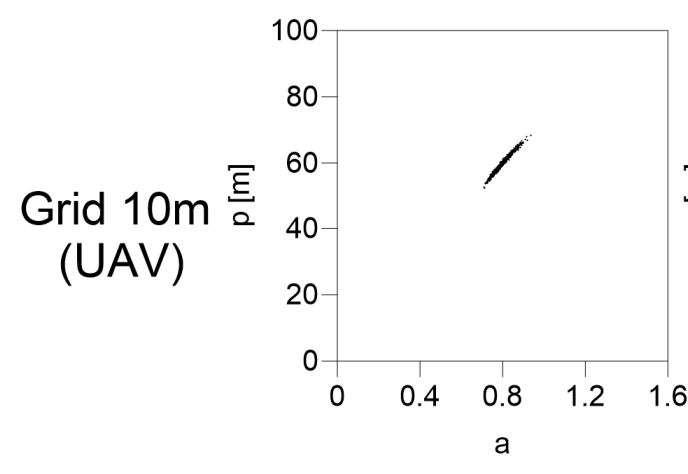

A

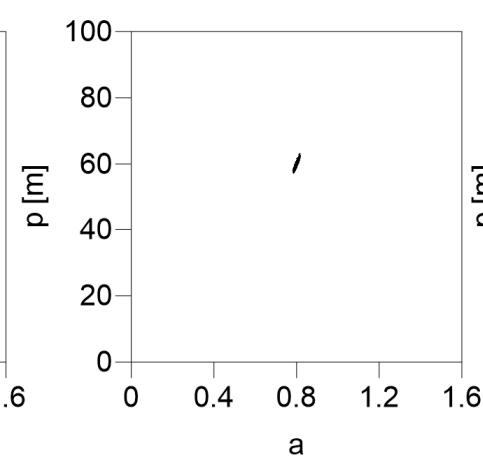

B

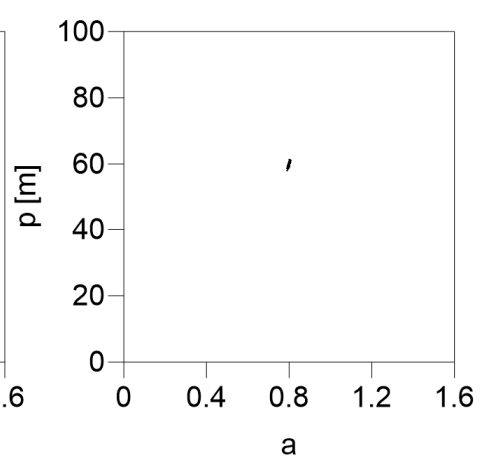

C

Fig. 5 Dependence of the $a$ and $p$ model parameter values.

Apart from the above-mentioned case of the WE line, the results of the estimations performed by using LPU and MC are convergent (Fig. 3). This proves the sufficient accuracy of approximating the parameter uncertainty by using the first derivatives of the values determined on a numerical basis. The dispersion of the uncertainty estimation (uncertainty of the uncertainty) of the parameter values using the LPU is lower by approximately one order of magnitude from the estimated value of uncertainty.

In most cases, the values of uncertainty decrease along with the exploitation progress. The increase in the uncertainty of determining the parameters $\tan \beta$ and $p$ along with exploitation progress for the NS line and parameter $\tan \beta$ for the NS/WE lines is 


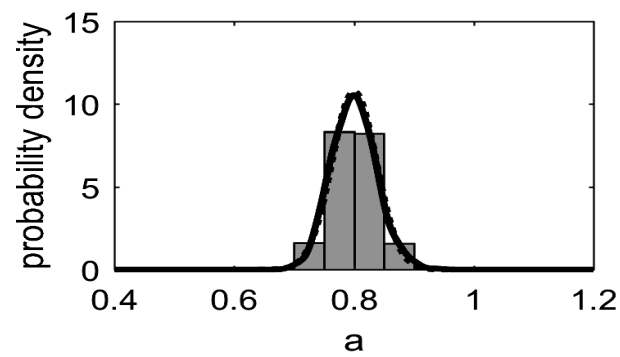

(a)

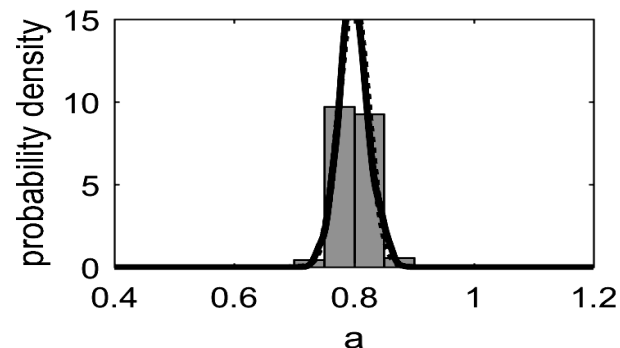

(c)

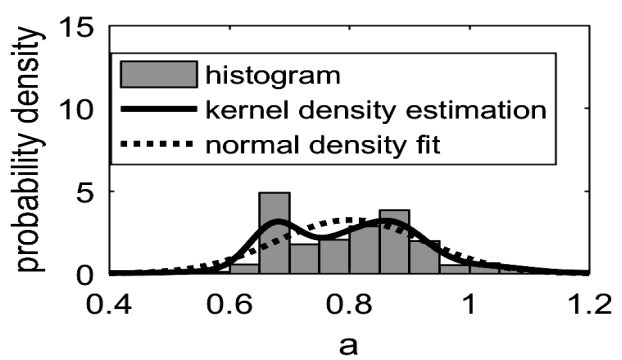

(b)

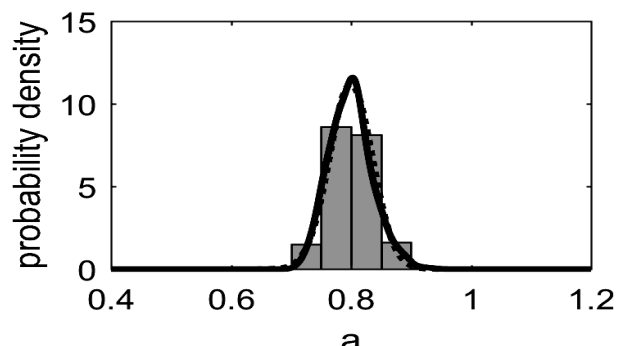

(d)

Fig. 6 The exemplary histograms and marginal distributions of exploitation coefficient a modelled by using the kernel smoothing function as well as their approximations with normal distribution: (a) NS line, (b) WE line, (c) NS / WE lines, (d) 10 m grid.

related to the increased value of the random factor of subsidences with a minor increase of the systematic factor. This ratio is crucial for the values of uncertainty of the determined parameters.

One of important features of the applied deformation model is the high correlation between the $a$ and $p$ parameters, in particular at the first stage (A) of the exploitation progress. The values of the correlation between the parameters are presented in Figure 4. Upon subsequent stages of exploitation, this correlation drops, although its general rate remains relatively high. For the remaining correlations, their change in value takes different courses along with the exploitation progress. In the case of parameters determined on the basis of data from the WE line and grid, the average estimations of the value of the correlation between the parameters are overstated compared with the values of these correlations determined by using the simulation.

Further insight in the correlation between the $a$ and $p$ parameters is revealed by the results of the conducted simulations presented in Figure 5. The graph illustrates the correlation between these model parameters. Each point on the graph corresponds to a single fit of the model (parameters) in each set of noisy subsidence values.

The simulation method enables the model parameter distributions to be modelled. The exemplary one-dimensional (marginal) distributions of parameters for the exploitation stage $\mathrm{A}$ is presented in Figure 6. Combined modelling of the model parameters - e.g. by using the kernel smoothing function estimate - is also possible. This creates wide opportunities for interpreting the results and applying the estimated parameter distributions while modelling the uncertainties of deformation indicator predictions.
In practice, however, trust in the modelled parameter distributions should be limited, since these are based on the assumed distribution of subsidence noises, which is only an approximation of their actual distribution. In addition, the Knothe model, despite its usefulness, is not error-free. For example, it does not consider the variance of parameters in space. Its underlying assumptions are only certain simplifications, thus excessively in-depth analysis and trust in the obtained distributions of parameters would be unwise.

The specific case of the WE line (stage A), in which a significant discrepancy in the estimation of uncertainty between two methods was revealed, relates to the unusually high value of the correlation between the $a$ and $p$ parameters. In such a case, the linear approximation (by estimating the uncertainty by using first derivatives) significantly overstates the parameter uncertainties. This is illustrated in Figure 7. The average value of the estimation of the coefficient of correlation between the $a$ and $p$ parameters by using LPU is 0.9998 with a standard deviation of 0.0004 , whereas the value of this coefficient estimated by using simulation amounts to 0.9666 . Thus, the $a$ and $p$ parameters are strongly correlated, provided that, in the specific presence of parameters optimal for a given noise distribution, this correlation is very close to the linear one. In such a case, the approximation of the covariance value, and therefore the coefficient of the correlation, by using the first derivatives causes some (minor) exaggeration of its value. In normal cases, this has no significant impact on the estimated values of parameter uncertainties. In this one, however, the (true) value of the coefficient of the correlation (covariance) estimated by the simulation is high enough that even its slightest 

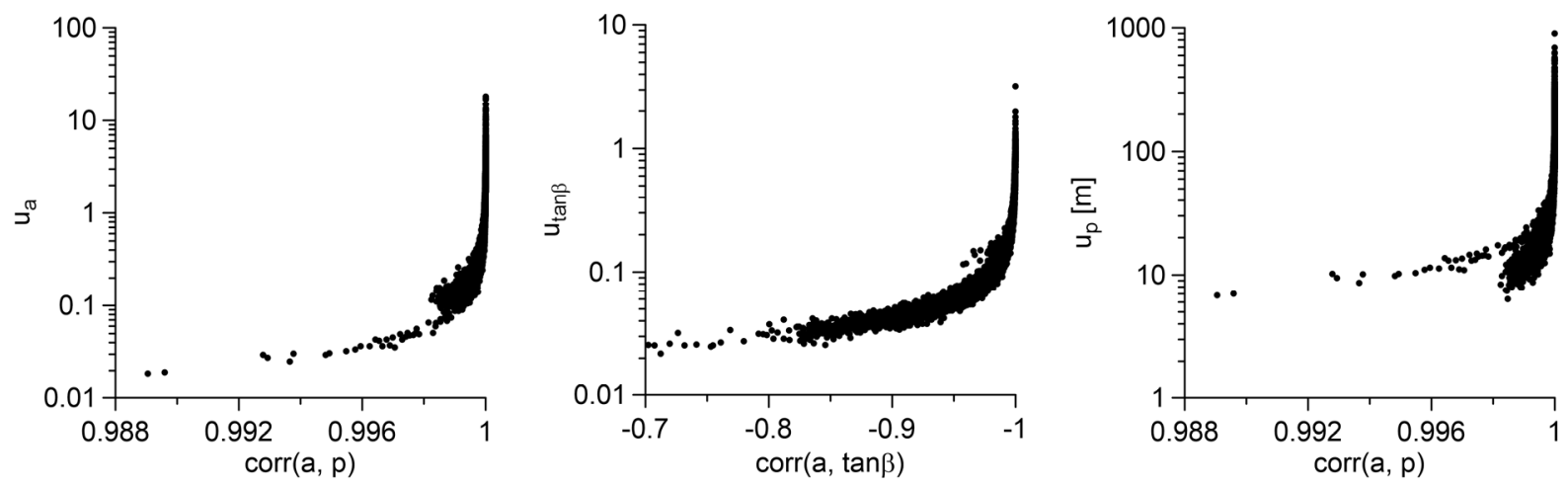

Fig. 7 Dependence between uncertainties estimated by the LPU and correlations between parameters estimated with this law for exploitation stage A for the WE line.

overstatement makes the value of the coefficient of the correlation increase even to the value of one (complete linear correlation).

According to the analysis (Gruszczyński et al., 2018) of the matrix diagonal $\left(A^{T} A\right)^{-1}$, when the correlation between the parameters moves towards one, the denominator of variance estimation (and thus uncertainty) of parameters moves towards zero. This means that the estimated value of the parameter variance moves simultaneously towards infinity.

Comparison of the uncertainties of the model parameters determined by using (simulated) data by UAV in the grid (with the results from the line) indicates the high potential of this measurement method. Despite the large (assumed) error in determining the subsidence of a single point, the uncertainties of the determined parameters are relatively low due to high data redundancy (Fig. 3). One should consider that the calculations used a $10 \mathrm{~m}$ grid and the actual potential of this method in terms of measurement data density are much greater (Gruszczyński et al., 2017). In practice, the ground sample distance corresponds to a few centimeters and obtaining the subsidence grid of a side of $0.25 \mathrm{~m} \div 1.0 \mathrm{~m}$ should not be problematic even after de-noising the measurement data. If the grid density increases, the uncertainty of determining the parameters will decrease linearly along with grid side length. This means that the uncertainties of parameters determined by using this measurement method will be, after the first stage of exploitation (A), of the following order of magnitude: $U_{a} \approx 0.003$, $U_{\tan \beta} \approx 0.002, U_{p} \approx 0.2 \mathrm{~m}$.

Unfortunately, each reduction of grid side length by half translates into a fourfold increase in the number of analyzed points, which significantly expands the time necessary for the calculations. In practice, taking the current capacity and continuous development of computers into consideration, this issue is of a technical rather than scientific nature. The elimination of systematic height errors determined by
UAV method and related, for instance, to seasonal vegetation growth is much more important.

\section{CONCLUSIONS}

On the basis of the studies conducted, the following synthetic conclusions have been drawn:

1. The values of parameter uncertainties determined by using LPU are in general convergent with the values determined by simulation. Due to the time required for computations, performing estimations by using the law of propagation of uncertainty is more favourable compared with Monte Carlo simulation.

2. Dispersion (uncertainty) of the determined uncertainties of parameters is much lower compared with the uncertainty of parameters.

3. Uncertainties of parameters determined by using LPU may be significantly overstated when the estimated values of the correlation between the parameters approach one. In such a case, the correct estimation of the uncertainties can be obtained by using the Mote Carlo simulation method.

4. The determination of parameters on the basis of data obtained in a dense and regular grid - e.g. by using close-range remote sensing by UAV - has great potential. This results from high data redundancy despite the significant uncertainty of determining the height (and subsidence) of single point.

\section{ACKNOWLEDGMENTS}

This work was supported by the statutory research WGGiIS' AGH no. 11.11.150.195 (Department of the Protection of Mining Areas, Geoinformatics and Mining Surveying) and by the Ministry of Science and Higher Education under the grant no. 15.11.150.390.

\section{REFERENCES}

Byrnes, R.: 2003, Case studies in the application of influence functions to visualising surface subsidence. 4th Australasian Coal Operator's Conference. Wollongong. 
Doležalová, H., Kajzar, V., Souček, K. and Staš L.: 2009, Evaluation of mining subsidence using GPS data. Acta Geodyn. Geomater., 6, 3, 359-367.

Ghabraie, B., Ren, G., Barbato, J. and Smith, J.V.: 2017, A predictive methodology for multi-seam mining induced subsidence. International Journal of Rock Mechanics and Mining Sciences, 93, 280-294. DOI: 10.1016/j.jirmms.2017.02.003

Gruszczyński, W., Niedojadło Z. and Mrocheń D.: 2018, Influence of model parameter uncertainties on forecasted subsidences. Acta Geodyn. Geomater., 15, 3, 211-228. DOI: 10.13168/AGG.2018.0016

Gruszczyński, W., Matwij, W., and Ćwiąkała, P.: 2017, Comparison of low-altitude UAV photogrammetry with terrestrial laser scanning as data-source methods for terrain covered in low vegetation. ISPRS Journal of Photogrammetry and Remote Sensing, 126, 168179. DOI: $10.1016 /$ j.isprsjprs.2017.02.015

Gruszczyński, W.: 2007, Application of neural networks for prediction of deformations modelling (Ph.D. thesis). AGH-UST, Cracow, (in Polish).

Hejmanowski, R. and Malinowska, A.A.: 2016, Significance of the uncertainty level for the modeling of ground deformation ranges, level for the modeling of ground deformation ranges. International Journal of Rock Mechanics and Mining Sciences, 83, 140-148. DOI: 10.1016/j.ijrmms.2015.12.019

Jiang, S., and Jiang, W.: 2017, Efficient structure from motion for oblique UAV images based on maximal spanning tree expansion. ISPRS Journal of Photogrammetry and Remote Sensing, 132, 140-161. DOI: 10.1016/j.isprsjprs.2017.09.004

Karmis, M., Agioutantis, Z. and Jarosz, A.: 1990, Recent developments in the application of the influence function method for ground movement predictions in the US. Mining Science and Technology, 10, 3, 233245. DOI: 10.1016/0167-9031(90)90439-Y

Karmis, M., Jarosz, A., Schilizzi, P. and Agioutantis, Z.: 1987, Surface deformation characteristics above undermined areas: Experience from the Eastern United States. Civil Engineering Transactions, 29, 2, $106-114$.

Knothe, S.: 1957, Observations of surface movements under influence of mining and their theoretical interpretation. In: Proceeding European Congress on Ground Movement, Leeds, 210-218.

Kowalski, A.: 2007, Transient mining surface deformations in the prediction accuracy aspect. Central Mining Institute, Pap No. 871, Katowice, (in Polish).

Kwinta A.: 2011, Application of the least square method in determination of the Knothe deformation prediction theory parameters. Arch Min Sci, 56, 2, 319-329.

Kwinta A.: 2010, Estimation of errors of deformation indicators based on the accuracy of the parameters of Knothe's theory. Przegląd Górniczy, 11, 39-45, (in Polish).
Lizarazo, I., Angulo, V. and Rodriguez, J.: 2017, Automatic mapping of land surface elevation changes from UAV-based imagery. International Journal of Remote Sensing, 38, No. 8-10, 2603-2622. DOI: $10.1080 / 01431161.2016 .1278313$

Lucieer A., Turner D., King D.H. and Robinson S.A.: 2014, Using an Unmanned Aerial Vehicle (UAV) to capture micro-topography of Antarctic moss beds. International Journal of Applied Earth Observation and Geoinformation, No. 27, 53-62. DOI: 10.1016/j.jag.2013.05.011

Luo, Y. and Cheng, J.W.: 2009, An influence function method based subsidence prediction program for longwall mining operations in inclined coal seams. Mining Science and Technology, 19, 592-598. DOI: 10.1016/S1674-5264(09)60110-1

Mierzejowska, A.: 2014, Modeling of the influence of average error values of the assumed parameter values in the theory of the influence on the average error of prediction of subsidence, inclinations and curvatures of the mining area. Przegląd Górniczy, 8, 171-176, (in Polish).

Ren, G., Li, G. and Kulessa, M.: 2014, Application of a generalized influence function method for subsidence prediction in multi-seam longwall extraction. Geotechnical and Geological Engineering, 32, 4, 1123-1131. DOI: 10.1007/s10706-014-9787-y

Stoch, T.: 2005, Impact of mining and geological conditions of mining extraction on randomness of displacement and surface deformation (Ph.D. thesis). Cracow: AGH-UST, (unpublished, in Polish).

Tajduś, K.: 2009, New method for determining the elastic parameters of rock mass layers in the region of underground mining influence. International Journal of Rock Mechanics and Mining Sciences, 46, 8, 12961305. DOI: 10.1016/j.ijrmms.2009.04.006

Tsai, C.H. and Lin, Y.C.: 2017, An accelerated image matching technique for UAV orthoimage registration. ISPRS Journal of Photogrammetry and Remote Sensing, No. 128, 130-145. DOI: 10.1016/j.isprsjprs.2017.03.017

Whittaker, B.N. and Reddish, D.J.: 1989, Subsidence: Occurrence, Prediction and Control. Nottingham, UK, $528 \mathrm{pp}$. 